\title{
Effects of Dexmedetomidine Infusion During Sevoflurane Anesthesia on Otoacoustic Emissions
}

\author{
Mehmet İlhan Şahin ${ }^{1}$, Alperen Vural ${ }^{1}$, Aynur Akın², İbrahim Ketenci ${ }^{1}$, and Yaşar Ünlü ${ }^{1}$ \\ ${ }^{1}$ Departments of Otolaryngology, ${ }^{2}$ Anesthesia, Erciyes University Faculty of Medicine, Kayseri, Turkey
}

Received August 13, 2018

Revised September 24, 2018

Accepted October 28, 2018

Address for correspondence

Mehmet İlhan Şahin, MD

Department of Otolaryngology,

Erciyes University Faculty of Medicine, Erciyes Üniversitesi

Tıp Fakültesi Gevher Nesibe

Hastanesi KBB AD Talas Yolu,

Melikgazi, Kayseri 38039, Turkey

Tel $+903522076666($ ext 21457)

Fax +90352 2076600

E-mail misahin@erciyes.edu.tr
Background and Objectives: Knowing the ototoxic potential of the agents used in medical treatments is important for the protection of hearing. Although we have knowledge regarding some effects of dexmedetomidine, which is an anesthetic-sparing drug, its influence over the hearing system has never been studied and is obscure yet. The aim of this study is to determine the effects of intravenous dexmedetomidine application during sevoflurane anesthesia on otoacoustic emissions (OAEs). Subjects and Methods: This prospective randomized study was performed on 60 patients (34 male, 26 female, mean age: $30.6 \pm 9.2$ years) who were scheduled for an elective surgery under general anesthesia and the patients were enrolled and randomly divided into 2 groups. They received dexmedetomidine (Group D) or Saline (Group S) infusion during a standardized Sevoflurane anesthesia. Transient and distortion product OAEs were measured preoperatively and postoperatively (24th hour). OAE results were compared within and between groups. Results: In group D postoperative OAEs were lower than preoperative OAEs and postoperative levels of group $\mathrm{S}$, especially at low frequencies $(p<0.05)$. Conclusions: Dexmedetomidine infusion affects the micromechanical function of cochlea especially in the low-frequency region. Dexmedetomidine should be carefully used during general anesthesia to avoid its probable harmful effects on cochlear micromechanics.

J Audiol Otol 2019;23(2):89-95

KEY WORDS: Dexmedetomidine · Adrenergic alpha-2 receptor agonists · Otoacoustic emissions $\cdot$ Sevoflurane $\cdot$ Inhalational anesthetics.

\section{Introduction}

Knowing the ototoxical potential of the agents used in medical and surgical treatments is important for protection of hearing. It is well known that ototoxicity usually starts at outer hair cells of cochlea so micromechanical function of cochlea is decreased before hearing function is lost at any early damage. This may not be detected by pure tone audiometry (PTA) but can be diagnosed by otoacoustic emission (OAE) measurements [1].

Effects of several general anesthetic agents on cochlear micromechanics have been researched for last decades. Some controversial OAE results have been reported because of the changes of middle ear pressure (MEP) and hemodynamia,

This is an Open Access article distributed under the terms of the Creative Commons Attribution Non-Commercial License (https://creativecommons.org/licenses/by-nc/4.0/) which permits unrestricted non-commercial use, distribution, and reproduction in any medium, provided the original work is properly cited. which may affect measurements besides agents' direct pharmacological effects on cochlear micromechanics [2,3]. However sevoflurane, an inhalational anesthetic, seems to be safe in terms of ototoxicity [4-6]. Despite knowing anesthetic drug's safety without knowledge of anaesthetic-sparing drugs' effects general anesthesia protocols should not be considered as safe.

One of the anaesthetic-sparing drug dexmedetomidine is a selective $\alpha 2$ adrenergic agonist. It is getting popular because of some beneficial effects such as sedation and anxiolysis [7]. $\alpha 2$-agonists have various effects especially on cardiovascular and central nervous systems. They decrease blood pressure and heart rate by centrally mediated sympatholytic effects. On the other hand, they induce peripheral vasoconstriction by directly activating vascular smooth muscle $\alpha 2$ receptors $[8,9]$. Dexmedetomidine was reported to cause a decrease in cerebral blood flow in dogs during anaesthesia both with halothane and isoflurane, morever it has been shown to be neuroprotec- 
tive in the animal models of brain ischaemia [10,11]. Although we have knowledge about some effects of dexmedetomidine, its influence over hearing system has never been studied and is obscure yet.

The aim of this study is to determine the effects of intravenous dexmedetomidine application during sevoflurane anaesthesia on cochlea by the way of OAE measurements.

\section{Subjects and Methods}

\section{Patients}

After obtained approval of the Institutional Ethics Committee (2009 Sept 3, approval no. 2009/35) patients who were scheduled for an elective surgery under general anesthesia and classified as ASA I according to the American Society of Anesthesiologists classification of physical status were enrolled. Patients with any ear disease or a history of an otological surgery were excluded. Chronic drug addicts, smokers, and patients with any type of systemic disease were also excluded.

Otoscopy, PTA (Audiometer AC40, Interacoustics, Assens, Denmark) and tympanometry tests (Impedance Audiometer AZ26, Interacoustics) were performed before the operation and results were classified as normal or abnormal. Defined normality criteria for an ear were to have a normal external canal and drum, with air conduction thresholds under $20 \mathrm{~dB}$ HL at $500,1,000,2,000 \mathrm{~Hz}$ and under $30 \mathrm{~dB} \mathrm{HL}$ at all frequencies, air bone gap under $10 \mathrm{~dB}$ at all frequencies, and tympanometric peak pressure between -100 and +100 daPa and normal acoustic reflex. Only one ear of patients which were identified as normal were included to the study. If both ears were "normal" relatively better ear in terms of hearing and pressure was included. If both ears were equal, the opposite ear of the surgical side for neck surgeries, and randomly chosen ear for the other surgeries was included.

All patients were asked to fill out informed consent form and were divided in two groups (Group S and D) randomly by coin flipping preoperatively.

PTA and tympanometry tests were performed once again at postoperative day 1 and patients whose perviously included ear did not meet normality criterias were excluded. Recordings of 60 ears of 60 patients [34 male, 26 female, aged $30.6 \pm 9.2$ years (minimum 18, maximum 50)] were analyzed at the end of the study.

\section{Anesthesia protocol in groups}

Premedication wasn't administrated before the operation. In the operation room baseline measurements of systolic, diastolic and mean arterial blood pressure (MAP), heart rate and peripheral oxygen saturation by pulse oximeter $\left(\mathrm{SpO}_{2}\right)$ and body temperature levels were recorded. All these parameters were monitored automatically during the operation. All anesthetics were administered by the same anesthesiologists with a standardized protocol for induction and maintenance. All patients recieved $1.5 \mu \mathrm{g} / \mathrm{kg}$ fentanyl, $5-6 \mathrm{mg} / \mathrm{kg}$ thiopental sodium and $0.6 \mathrm{mg} / \mathrm{kg}$ rocuronium bromide intravenously for induction of anesthesia. Anesthesia was maintained with sevoflurane $1.5-2 \%$ in $50 \% \mathrm{~N}_{2} \mathrm{O}$ and $50 \%$ oxygen with controlled ventilation, resulting in an end-tidal $\mathrm{CO}_{2}$ of $4.6 \pm 0.5$ $\mathrm{kPa}(35 \pm 4 \mathrm{~mm} \mathrm{Hg})$. Also for maintenance of neuromuscular blocade rocuronium bromide $(10 \mathrm{mg})$ was administrated intravenously.

After induction of anesthesia, patients received dexmedetomidine or saline i.v. during whole maintenance of anesthesia. Patients in dexmedetomidine group (Group D) recieved $1 \mu \mathrm{g}$ / kg dexmedetomidine (Precedex, Abbott Labs., North Chicago, IL, USA) as initial loading dose over 10 minutes and infusion was also applied with a dose of $1 \mu \mathrm{g} / \mathrm{kg}$ per hour in $100 \mathrm{~mL}$ saline. Patients in saline group (Group S) recieved $100 \mathrm{~mL}$ saline i.v. over 10 minutes and infusion was applied with a dose of $1 \mathrm{~mL} / \mathrm{kg}$ per hour. The otolaryngologist who have recorded the measurements was blinded to the drug.

Duration of the operation (period between beginning of the induction and ending of the maintenance of general anesthesia) was recorded.

\section{Measurements of OAEs}

Measurements of OAE (Capella Cochlear Emissions Analyzer, GN Otometrics A/S, Taastrup, Denmark) were repeated at a silent room preoperatively and postoperatively ( 24 hours after the operation) and patients were instructed to be immobile in sitting position during recordings.

Transient evoked otoacoustic emissions (TOAEs) were measured by a fast screen mode using stimuli consisting a click of $40 \mu$ s duration and $75 \mathrm{~dB}$ SPL level. The click rate was 50/ $\mathrm{s}$, and poststimulus analysis was in the range of 3-12.5 ms. Criteria to discontinue the tests were 2,080 scans. Signal to noise $(\mathrm{S} / \mathrm{N})$ ratio was measured and recorded as TOAE response at 1,000, 1,500, 2,000, 3,000, and 4,000 Hz.

Distortion product otoacoustic emissions (DpOAEs) were measured using a series of simultaneous stimulus whose ratio of frequencies (f2/f1) was 1.21, and intensities (L1, L2) were $65 \mathrm{~dB}$ SPL and $55 \mathrm{~dB}$ SPL respectively. Schematic depiction of DpOAEs (DP-gram) was obtained by the emissions at $2 \mathrm{f} 1-\mathrm{f} 2$ and $\mathrm{S} / \mathrm{N}$ ratios were recorded at a range of frequencies $500-8,000 \mathrm{~Hz}$. The criteria to stop the test was 1,000 scanning to be done. 


\section{Other measurements}

Systolic and diastolic blood pressures were measured noninvasively just before preoperative and postoperative OAE measurements and MAPs were recorded. Intraoperative mean blood pressure measurements were recorded every $15 \mathrm{~min}$ utes from the beginning of induction of anesthesia to 120th minute.

Body temperature was measured at the axilla just before preoperative and postoperative OAE measurements using electronic thermometer. Intraoperative body temperature measurement and monitorization was done by skin probe, and recorded with OAE measurement simultaneously.

\section{Statistical analysis}

To analyse the normality of the distributions of data the Shapiro-Wilk test was used. Data that were normally distributed were defined by mean and standard deviation, and which were not normally distributed were defined by median (minimum-maximum). To determine the significance of the change of the OAE measurements within the groups paired t-test was used for data which were normally distrubuted. The Wilcoxon signed rank test was used for the data that does not show normal distribution. To compare the mean arterial pressure, body temperature and OAE measurements between groups independent samples t-test was used for data which were normally distrubuted and Mann-Whitney $U$ test was used for data that were not normally distrubuted. $p<0.05$ was accepted as the level of significance.

\section{Results}

Randomly assigned 30 patients participated in each group. Age, gender, numbers and mean durations of the performed operations were similar between groups (Table 1).

There was no statistically significant difference between the groups in terms of body temperature $(p>0.05)$ (Table 1$)$. Patients' MAPs were not significantly different between groups preoperatively ( $p=0.690)$ and postoperatively $(p=0.608)$. At the beginning of the operations (intraoperative $0,15,30 \mathrm{~min}$ ) blood pressure levels were similar between the groups as well $(p>0.05)$. However, blood pressure levels at intraoperative $45,60,75,90,105$, and $120 \mathrm{~min}$ were significantly lower in group D than group S $(p<0.001)$. But arterial pressures never decreased under the hypotensive level (MAP $<60 \mathrm{~mm} \mathrm{Hg}$ ) (Fig. 1).

\section{Transient OAE results}

\section{Inter-group comparisons}

It was observed that the $\mathrm{S} / \mathrm{N}$ ratios were similar between two groups at all frequencies preoperatively $(p>0.05)$, but $\mathrm{S} /$ $\mathrm{N}$ ratio at the frequency of $3,000 \mathrm{~Hz}$ was significantly lower in the group $\mathrm{D}$ postoperatively $(p=0.023)$.

\section{Intra-group comparisons}

In the group $\mathrm{D}$, postoperative $\mathrm{S} / \mathrm{N}$ ratios at $1,000,1,500$, and $2,000 \mathrm{~Hz}$ were significantly lower than the preoperative levels $(p<0.001)$, whereas in the group $\mathrm{S}$ mean postoperative $\mathrm{S} / \mathrm{N}$ ratios were not statistically different as per the preoperative values at all frequencies ( $p>0.05)$ (Fig. 2).

Table 1. Comparison of the data of age, gender, number and durations of the performed operations and body temperature between 2 groups

\begin{tabular}{|c|c|c|c|}
\hline & Group S $(n=30)$ & Group D $(n=30)$ & p-value \\
\hline \multicolumn{4}{|l|}{ Age (years) } \\
\hline Mean $\pm S D$ & $30.57 \pm 8.99$ & $30.53 \pm 9.59$ & 0.989 \\
\hline \multicolumn{4}{|l|}{ Gender (n) } \\
\hline Male & 17 & 17 & 1 \\
\hline Female & 13 & 13 & 1 \\
\hline \multicolumn{4}{|l|}{ Performed operations (n) } \\
\hline Nasal & 22 & 20 & 0.373 \\
\hline Neck & 7 & 6 & 0.350 \\
\hline Oropharyngeal & 1 & 4 & 0.313 \\
\hline \multicolumn{4}{|c|}{ Durations of the operations (minutes) } \\
\hline Mean \pm SD & $117 \pm 67.70$ & $116.83 \pm 45.08$ & 0.991 \\
\hline \multicolumn{4}{|l|}{ Body Temperature $\left({ }^{\circ} \mathrm{C}\right)$} \\
\hline Preoperative (mean \pm SD) & $36.46 \pm 0.40$ & $36.43 \pm 0.36$ & 0.759 \\
\hline Intraoperative (mean \pm SD) & $36.31 \pm 0.27$ & $36.38 \pm 0.36$ & 0.360 \\
\hline Postoperative (mean \pm SD) & $36.70 \pm 0.34$ & $36.66 \pm 0.32$ & 0.612 \\
\hline
\end{tabular}




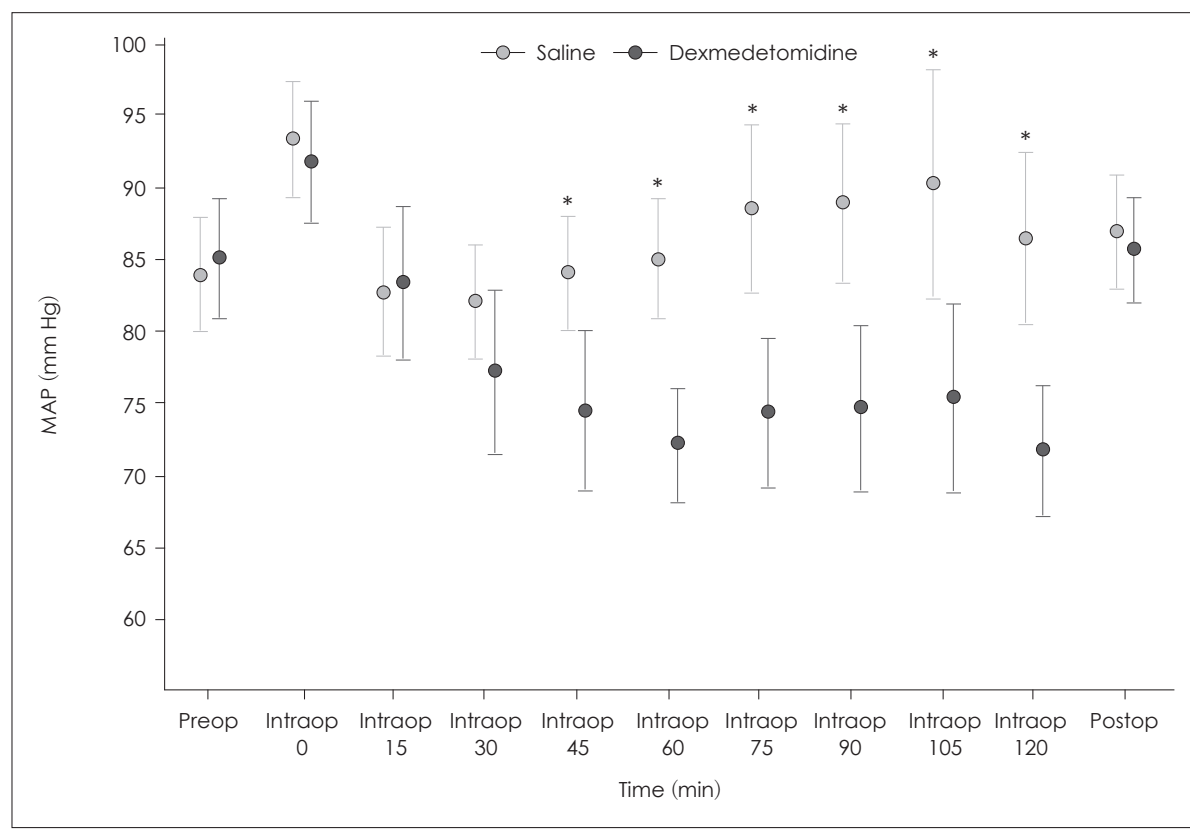

Fig. 1. Change of mean arterial pressure by time and comparison of 2 groups. *statistically significant difference between 2 groups, $p<0.001$. MAP: mean arterial blood pressure.

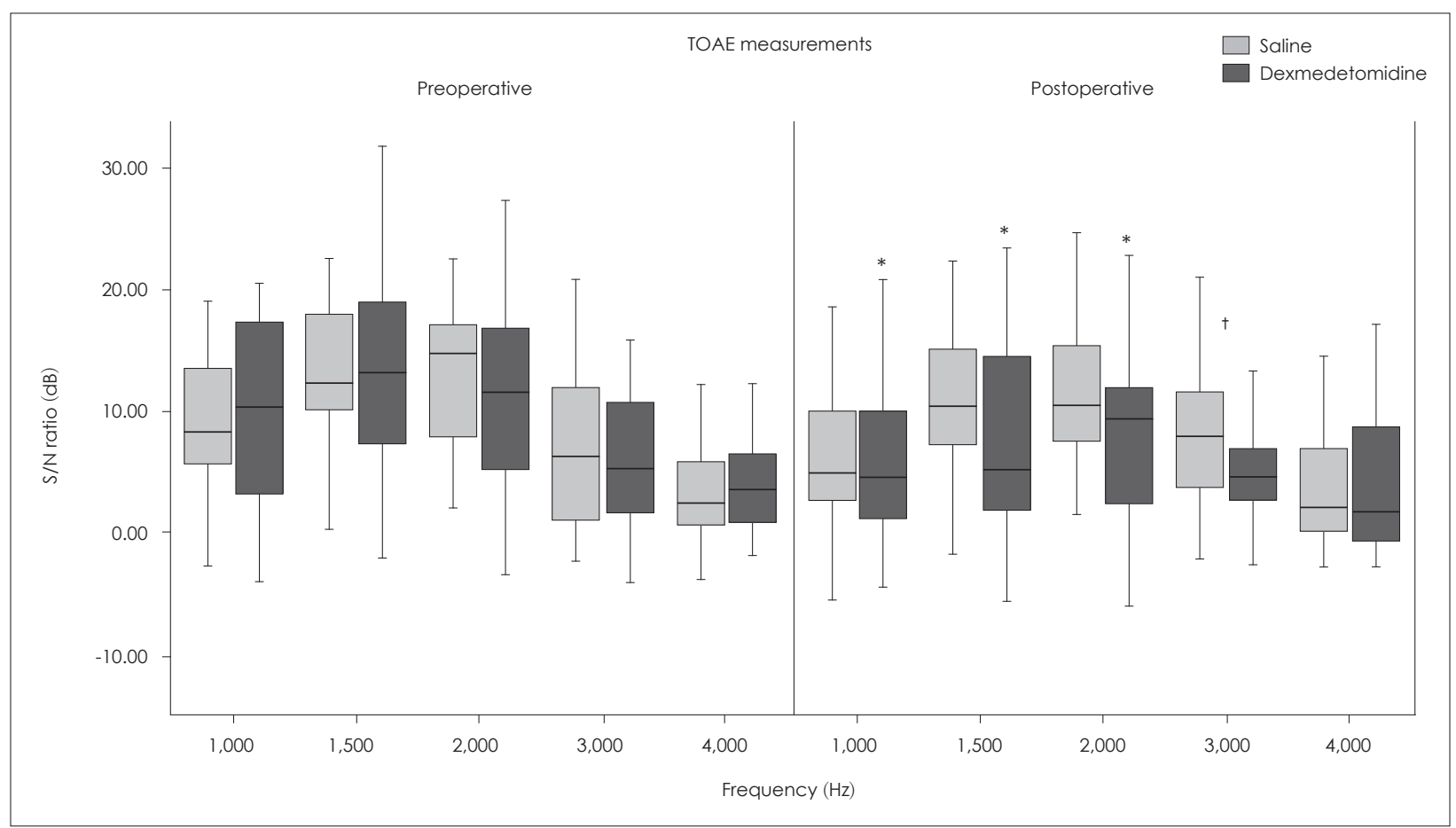

Fig. 2. TOAE results by time and comparison of 2 groups. *values that are significantly lower than preoperative level, $p<0.001$, ${ }^{\dagger}$ significant difference between groups, $p<0.05$. TOAE: transient otoacoustic emission.

\section{DpOAE results}

\section{Inter-group comparisons}

The analysis showed that the preoperative measures were similar at all frequencies $(p>0.05)$. The mean postoperative $\mathrm{S} / \mathrm{N}$ ratios were significantly lower in the group $\mathrm{D}$ than that of the group $\mathrm{S}$ at $500,750,1,000$, and $2,000 \mathrm{~Hz}(p<0.05)$ and they were not significantly different at $1,500,3,000,6,000$, and
$8,000 \mathrm{~Hz}$ between the two groups $(p>0.05)$.

\section{Intra-group comparisons}

In the group $\mathrm{D}$ mean postoperative $\mathrm{S} / \mathrm{N}$ ratios were lower than the preoperative ratios at all frequencies but this difference was not statistically significant only at $1,500 \mathrm{~Hz}$ ( $p<$ $0.05)$. However in the group $S$ there were no significant difference between preoperative and postoperative $\mathrm{S} / \mathrm{N}$ ratios at 


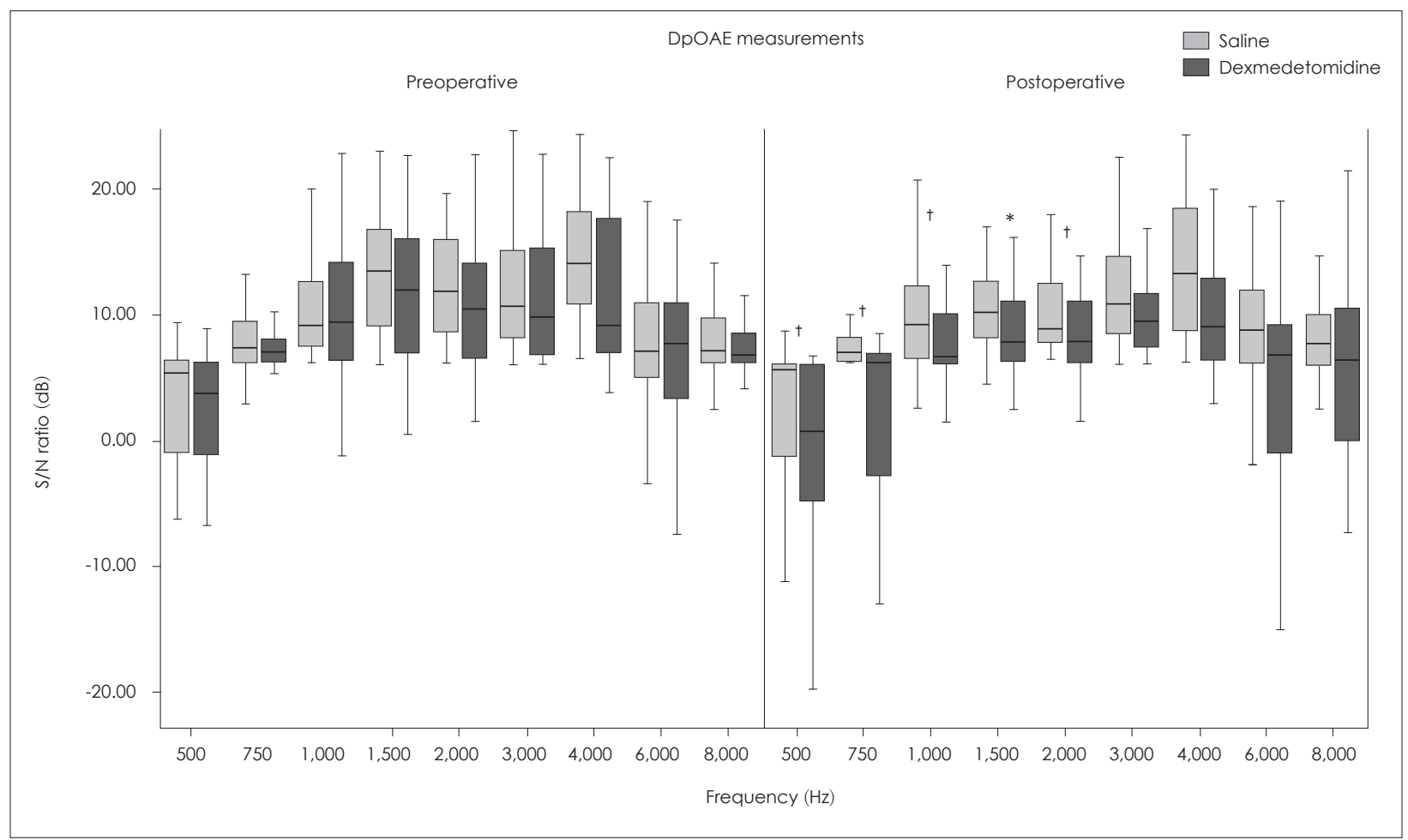

Fig. 3. Change of DpOAE results by time and comparison of 2 groups. *values that are significantly lower than preoperative level, $p<$ 0.05 , tsignificant difference between groups, $p<0.05$. DpOAE: distortion product otoacoustic emission.

all frequencies $(p>0.05)$ (Fig. 3).

\section{Discussion}

The effects of several agents used within general anesthesia protocols on hearing has been documented in the literature but dexmedetomidine's effect is unknown. We have found some significant differences in OAEs postoperatively between patients who received standard sevoflurane anesthesia protocol with and without dexmedetomidine infusion. Results confirmed safety of standard sevoflurane protocol in terms of damaging cochlear micromechanical function but i.v. dexmedetomidine infusion attracted suspicion.

The effect of anaesthetic agents on OAEs has been the focus of several studies throughout years. Hauser, et al. [12] mentioned that TOAE amplitudes decrease in patients who take enfluran, this decrease is more when $\mathrm{N}_{2} \mathrm{O}$ is added. It is emphasized that the decrease of the OAE level in anesthetic applications is a result of variations of pressure in the middle ear rather than the direct effect of the agent [12]. In fact, various studies revealed that alterations of MEP decrease OAEs especially at low frequencies [13-15]. It is known that the $\mathrm{N}_{2} \mathrm{O}$ increases the MEP, and sevoflurane may increase it during the application as well $[16,17]$. Both of these agents were involved in the anesthesia protocol which applied to all patients in this study. However, postoperative changes of OAEs were not related to MEP because it was proved to be in the normal ranges by tympanometry.

OAE variations resulting from anesthetic applications are not only related to variations of the MEP but also variations of the blood pressure and cochlear blood flow. It is known that hearing physiology and cochlea are sensitive to systemic blood pressure variations. There are studies which revealed that hypotension may lead to immediate or slow hearing loss, and the loss is apparent especially at the low frequency areas $[18,19]$. The findings of the experimental studies which indicate that DpOAE levels decrease only seconds after cochlear blood flow is stopped at the internal acoustic channel confirm the sensitivity of outer hair cells to ischemia [20,21]. In this study, although the MAPs of the dexmedetomidine group were significantly lower than that of the saline group intraoperatively, lower levels of postperative OAEs in the group D were not thought to be as a result of this, because patients' MAPs never decreased under the hypotensive level which is critical for tissue perfusion. On the other hand this may be related to cochlear blood flow. It was revealed that cochlear blood flow has own autoregulation [22]. Because of being a neurosensorial organ the blood flow autoregulation of cochlea is similar to cerebral blood flow autoregulation, and it should be considered that agents which damage cerebral autoregulation may 
also damage cochlear autoregulation. Dagal and Lam [23] and Albera, et al. [4] supports this consideration. Dagal and Lam [23] stated that sevoflurane protects cerebral autoregulation and Albera, et al. [4] showed that sevoflurane protects cochlear blood flow. Although there is no study in the literature that investigated the effects of dexmedetomidine on cochlear blood flow it was asserted in Ogawa's study [24] that dexmedetomidine damages cerebral blood flow autoregulation and decreases cerebral blood flow. It was also revealed that dexmedetomidine has a systemic peripheric vasoconstrictor effect $[25,26]$. Then it is conceivable to suggest that Dexmedetomidine very likely damages cochlear blood flow autoregulation and decreases cochlear blood flow.

In addition to MEP and cochlear blood flow some other factors which may affect OAE results were taken into consideration in this study. Since the effects of agents on cochlea might vary depending on dose, durations of the operations were recorded. Also body temperature was measured from the skin during the pre-, intra-, and postoperative OAE measures, and the normothermic process has been confirmed. Because it was stated in the empirical and clinical studies in the literature that body temperature can affect OAEs, especially hypothermia can decrease the OAE amplitudes [27-29]. However no significant difference was found between two groups in terms of the mean duration of the operations and body temperature. Therefore, the differences between the postoperative OAE values of two groups were considered to be unrelated with the duration of the operations or body temperature of the patients.

The fact that postoperative OAE levels were not significantly different from preoperative levels for the patients in the group $\mathrm{S}$ reveal that sevoflurane anaesthesia did not have a permanent effect on cochlear micromechanics. These findings confirm the findings of Buyukkocak, et al.'s [5] clinical study which had also revealed no difference between postoperative and preoperative measures of PTA and TOAEs of the patients who were given sevoflurane anaesthesia. So it is considered that this agent could be reliably used in otorhinolaryngological surgeries in terms of ototoxicity.

Furthermore, it was found that i.v. dexmedetomidine application affects micromechanical function of cochlea especially at the low frequency region. In order to understand the reason of this effect clearly, further research is needed to clarify the effect of dexmedetomidine on cochlear blood flow. Herewith, dexmedetomidine should be carefully used during general anesthesia to avoid its possible harmful effects on cochlear micromechanics.

\section{Conflicts of interest}

The authors have no financial conflicts of interest.

\section{REFERENCES}

1) Pawlowski K. Anatomy and Physiology of the Cochlea. In: Ototoxicity (ed. Roland P, Rutka J). London: BC Decker Inc;2004. p.1-19.

2) Harel N, Kakigi A, Hirakawa H, Mount RJ, Harrison RV. The effects of anesthesia on otoacoustic emissions. Hear Res 1997;110:25-33.

3) Zheng Y, Ohyama K, Hozawa K, Wada H, Takasaka T. Effect of anesthetic agents and middle ear pressure application on distortion product otoacoustic emissions in the gerbil. Hear Res 1997;112:16774.

4) Albera R, Ferrero V, Canale A, De Siena L, Pallavicino F, Poli L. Cochlear blood flow modifications induced by anaesthetic drugs in middle ear surgery: comparison between sevoflurane and propofol. Acta Otolaryngol 2003;123:812-6.

5) Buyukkocak U, Kilic R, Arikan OK, Sert O, Datli F. Prospective randomized trial to determine whether inhalational anesthetics have any effects on hearing function. J Otolaryngol Head Neck Surg 2009; 38:495-500

6) Guven S, Tas A, Adali MK, Yagiz R, Alagol A, Uzun C, et al. Influence of anaesthetic agents on transient evoked otoacoustic emissions and stapedius reflex thresholds. J Laryngol Otol 2006;120:10-5.

7) Khan Z, Ferguson C, Jones R. Alpha-2 and imidazoline receptor agonists. Their pharmacology and therapeutic role. Anaesthesia 1999;54: 146-65.

8) Flacke JW. Alpha 2-adrenergic agonists in cardiovascular anesthesia. J Cardiothorac Vasc Anesth 1992;6:344-59.

9) Memiş D, Turan A, Karamanlıŏlu B, Şeker Ş, Pamukçu Z. Dexmedetomidine reduces rocuronium dose requirement in sevoflurane anaesthesia. Curr Anaesth Crit Care 2008;19:169-74.

10) Maier C, Steinberg GK, Sun GH, Zhi GT, Maze M. Neuroprotection by the alpha 2-adrenoreceptor agonist dexmedetomidine in a focal model of cerebral ischemia. Anesthesiology 1993;79:306-12.

11) Zornow MH, Fleischer JE, Scheller MS, Nakakimura K, Drummond JC. Dexmedetomidine, an $\alpha 2$-adrenergic agonist, decreases cerebral blood flow in the isoflurane-anesthetized dog. Anesth Analg 1990; 70:624-30.

12) Hauser R, Harris F, Probst R, Frei F. Influence of general anesthesia on transiently evoked otoacoustic emissions in humans. Ann Otol Rhinol Laryngol 1992;101:994-9.

13) Zhang M, Abbas PJ. Effects of middle ear pressure on otoacoustic emission measures. J Acoust Soc Am 1997;102(2 Pt 1):1032-7.

14) Sun XM, Shaver MD. Effects of negative middle ear pressure on distortion product otoacoustic emissions and application of a compensation procedure in humans. Ear Hear 2009;30:191-202.

15) Uchida $Y$, Ando F, Nakata S, Ueda H, Nakashima T, Niino N, et al. Distortion product otoacoustic emissions and tympanometric measurements in an adult population-based study. Auris Nasus Larynx 2006;33:397-401.

16) Nader ND, Simpson G, Reedy RL. Middle ear pressure changes after nitrous oxide anesthesia and its effect on postoperative nausea and vomiting. Laryngoscope 2004;114:883-6.

17) Ozturk O, Demiraran Y, Ilce Z, Kocaman B, Guclu E, Karaman E. Effects of sevoflurane and TIVA with propofol on middle ear pressure. Int J Pediatr Otorhinolaryngol 2006;70:1231-4.

18) Hansen S. Postural hypotension-cochleo-vestibular hypoxia-deafness. Acta Otolaryngol Suppl 1988;449:165-9.

19) Pirodda A, Ferri GG, Modugno GC, Gaddi A. Hypotension and sensorineural hearing loss: a possible correlation. Acta Otolaryngol 1999; 119:758-62.

20) Telischi FF, Stagner B, Widick MP, Balkany TJ, Lonsbury-Martin BL. Distortion-product otoacoustic emission monitoring of cochlear blood flow. Laryngoscope 1998;108:837-42. 
21) Mom T, Telischi FF, Martin GK, Lonsbury-Martin BL. Measuring the cochlear blood flow and distortion-product otoacoustic emissions during reversible cochlear ischemia: a rabbit model. Hear Res 1999; 133:40-52.

22) Brown JN, Nuttall AL. Autoregulation of cochlear blood flow in guinea pigs. Am J Physiol 1994;266(2 Pt 2):H458-67.

23) Dagal A, Lam AM. Cerebral autoregulation and anesthesia. Curr Opin Anaesthesiol 2009;22:547-52.

24) Ogawa Y, Iwasaki K, Aoki K, Kojima W, Kato J, Ogawa S. Dexmedetomidine weakens dynamic cerebral autoregulation as assessed by transfer function analysis and the thigh cuff method. Anesthesiology 2008;109:642-50.

25) Kawano T, Yamazaki F, Chi H, Kawahito S, Eguchi S. Dexmedetomidine directly inhibits vascular ATP-sensitive potassium channels.
Life Sci 2012;90:272-7.

26) Talke P, Lobo E, Brown R. Systemically administered alpha2-agonist-induced peripheral vasoconstriction in humans. Anesthesiology 2003;99:65-70.

27) Borin A, Cruz OL. Study of distortion-product otoacoustic emissions during hypothermia in humans. Braz J Otorhinolaryngol 2008; 74:401-9.

28) Ferber-Viart C, Savourey G, Garcia C, Duclaux R, Bittel J, Collet J. Influence of hyperthermia on cochlear micromechanical properties in humans. Hear Res 1995;91:202-7.

29) Seifert E, Brand K, Van de Flierdt K, Hahn M, Riebandt M, Lamprecht-Dinnesen $\mathrm{A}$. The influence of hypothermia on outer hair cells of the cochlea and its efferents. Br J Audiol 2001;35:87-98. 